\title{
Sero-prevalence and risk factors associated with toxoplasma gondii infection among pregnant women in Alexandria, Egypt
}

\author{
Hassan K. Bassiouny ${ }^{1}$, Nadia K. Soliman ${ }^{1}$, Sally El Tawab ${ }^{2 *}$, Safaa Mohamed Eassa1, \\ Amira Eissa ${ }^{1}$
}

\begin{abstract}
${ }^{1}$ Department of Pathology, High Institute of Public Health, Alexandria University, Alexandria, Egypt
${ }^{2}$ Department of Tropical Health, High Institute of Public Health, Alexandria, Egypt
\end{abstract}

Received: 06 October 2016

Accepted: 03 November 2016

\section{*Correspondence:}

Dr. Sally El Tawab,

E-mail: sally_eltawab@hotmail.com

Copyright: (c) the author(s), publisher and licensee Medip Academy. This is an open-access article distributed under the terms of the Creative Commons Attribution Non-Commercial License, which permits unrestricted non-commercial use, distribution, and reproduction in any medium, provided the original work is properly cited.

\begin{abstract}
Background: Toxoplasma gondii infection during pregnancy can result in fetal death, neonatal death or various congenital defects. This study aimed to estimate the seroprevalence of toxoplasma gondii infection using two different diagnostic tests, Enzyme-Linked Immunosorbent Assay (ELISA) versus Immunochromatographic assay (ICA) and to study the potential risk factors for acquiring infection in pregnant women attending antenatal care clinics in Alexandria, Egypt.

Methods: A cross sectional study, conducted between May 2015 and June 2016. The study was done in antenatal care centers of most districts of Alexandria Governorate, Egypt. 382 pregnant women, of a gestational age between 8-40 weeks were included in the study and were given pretested structured questionnaire to assess risk factors, which included: demographic, socio-economic data, kitchen hygiene and behavioral variables. Blood samples were taken and sera were divided into two parts; the first part was examined for anti-T.gondii IgG and IgM antibodies using rapid diagnostic test RDT kit, the other part tested by ELISA.

Results: The overall seroprevalence of T.gondii infection was $(11.3 \%)$ detected by RDT, significantly increased to $(57.9 \%)$ by ELISA test $(\mathrm{X} 2=5.3 ; \mathrm{p}=0.001)$. RDT had the sensitivity of $(15.8 \%)$, the specificity of $(95 \%)$, PPV of $(81.4 \%)$, NPV of $(45.1 \%)$, with overall diagnostic efficiency of (49.2\%). The association between T.gondii infection and the age of the pregnant women was found to be statistically significant (OR=2.84, 95\% $\mathrm{CI}=1.251-6.455)$.

Conclusions: The present study has documented a bad diagnostic performance of RDT in detection T.gondii in serum samples of infected pregnant women as compared to ELISA technique. Age is the only risk factor to be associated statistically with toxoplasma gondii infection.
\end{abstract}

Keywords: Antenatal care, Sero-prevalence, Toxoplasma gondii

\section{INTRODUCTION}

Toxoplasma gondii is an obligate intracellular protozoan pathogen that causes a disease called toxoplasmosis. Its medical importance remained unknown until 1939 when T. gondii was identified in tissues of a congenitally infected infant. ${ }^{1}$ The life cycle of $T$. gondii was described only in 1970, when it was discovered that its definitive hosts are members of the family Felidae, including domestic cats. Many warm-blooded animals including humans, cats and birds serve as intermediate hosts. ${ }^{2}$ The main routes of transmission of $T$. gondii in humans are ingestion of tissue cysts in undercooked or raw meat, or ingestion of vegetable, water, milk or anything contaminated with oocysts shed in feces of infected animals, beside, through placental transmission to the 
fetus. Also, it may be transmitted by blood transfusion and organ transplantation and may be through laboratory accidents. ${ }^{3}$

It infects about $30 \%$ of the population worldwide. ${ }^{4}$ The incidence of infection in humans and animals may vary in different parts of a country. The cause for these variations may be due to environmental conditions, food behavioral or anthropogenic, economic, social and cultural factors (hygienic practices, dietary habits, and cooking methods) as well as water quality and sanitation.

About 10-20\% of those with an acute infection will have enlarged lymph nodes in the cervical and inguinal region as well as flu-like symptoms (fever, headache, muscle pain). ${ }^{5}$ Focal areas of necrosis may develop in many organs. T. gondii does not produce a toxin; necrosis is caused by intracellular multiplication of tachyzoites which destroy the host cells. ${ }^{6}$ The infection is generally self-limited and the symptoms usually resolve in a few weeks or months. T. gondii during pregnancy can result in fetal death, neonatal death or various congenital defects, e.g. hydrocephalus, central nervous system abnormalities and chorioretinitis. ${ }^{7}$ The severity of congenital infection is inversely proportional to the transmission rate. Hence maternal infection acquired in the first trimester has a low transmission rate of 10-15\% and a severe infection outcome, whereas in infection during the third trimester, the transmission rate increases to about $68 \%$ while severity of the infection outcome is drastically reduced. ${ }^{8}$

In countries such as France, Austria, Brazil and Columbia, in-depth studies on congenital transmission of toxoplasmosis led to the formulation of policy to routinely screen pregnant women as part of their antenatal care and this has proven to greatly reduce the rate of the congenital form of the disease. ${ }^{9}$

The objective of the study was to estimate the seroprevalence of toxoplasma gondii infection using two different diagnostic tests, Enzyme-Linked Immunosorbent Assay (ELISA) versus Immunochromatographic assay (ICA) and to study the potential risk factors for acquiring infection in pregnant women attending antenatal care clinics in Alexandria, Egypt.

\section{METHODS}

This cross -sectional study was approved by the Ethical Review Committee of the High Institute of Public Health (HIPH), Alexandria University. The required data and blood samples were collected after a written informed consent from all participating pregnant women. The study was conducted between May 2015 and June 2016. The study was done in some antenatal care centres of most districts of Alexandria Governorate, its geographical coordinates of $31^{\circ} 12^{\prime} \mathrm{N}, 29^{\circ} 55^{\prime} \mathrm{E}$. The randomly selected antenatal care centers were two centers in El-
Ibrahimia and Moharem-Bey which belong to Alexandria Family Planning Association (AFPA). In addition to the health unit of Ezbet Danna and two private obstetrics clinic in Ezbet Skina and in El-Asafra. Collected samples were sent to Parasitology laboratory, Tropical Health Department, HIPH, Alexandria University.

The sample size (n) was calculated using the statistical formula:

$$
\mathrm{n}=\mathrm{Z} 2 \mathrm{p}(1-\mathrm{p}) / \mathrm{d} 2
$$

where $n=$ sample size, $Z=1.96$ at $95 \%$ confidence interval (CI), $\mathrm{P}$ is the expected prevalence $(46.5 \%)$, and $\mathrm{d}$ is the desired precision or margin of error $(5 \%)$. The required sample size was computed to be 382 pregnant women. ${ }^{10,11}$

All participating 382 pregnant women, of a gestational age between 8-40 weeks were a given pretested structured questionnaire to assess risk factors, which included: demographic, socio-economic data, kitchen hygiene and behavioral variables. The questionnaire included questions on age, place of residence, degree of education, occupation and obstetric history (spontaneous abortion, stillbirth, preterm delivery, and congenital anomalies). Besides, the medical history, and relevant clinical information as diabetes mellitus (DM), hypertension, Lymphadenopathy, fever, rash, edema etc. In addition, habits of consumption of under cooked meat, exposure to cats (pet cat ownership) or other animals, eating habits, and drinking water sources etc... Guidance on T.gondii infection was provided with special emphasis on ways of preventing infection.

From each participant about $4 \mathrm{ml}$. of venous blood were drawn in plain, pre labeled, dry clean test tubes and transported from antenatal care clinics to Parasitology laboratory, HIPH. All samples were left to coagulate for 30 minutes, and then separated by centrifugation at 3000 rpm for 4 minutes to obtain sera. Sera were divided into two parts; the first part was examined for anti- T.gondii IgG and IgM antibodies using rapid diagnostic test RDT kit (EUGENE TOXO IgG / IgM Rapid Test, Shanghai Eugene Biotech Co., Ltd, China). The other part was stored at $-20^{\circ} \mathrm{C}$ till serological tests were done for anti T.gondii IgG and IgM antibodies using ELISA test kit (Pre Check, Inc, USA). The two techniques were done following the manufacturer's instructions.

Interpretation of RDT results was through the absence of any color in both IgM line (M) and $\operatorname{IgG}$ line $(\mathrm{G})$ indicates that no $\operatorname{IgG}$ or $\operatorname{IgM}$ antibody to $\mathrm{T}$. gondii is detected in the specimen so the result is negative. Positive results: IgG positive: two colored lines, the control line $(\mathrm{C})$ and IgG line $(\mathrm{G})$ appear on the test strip. IgM positive: two colored lines, the control line $(\mathrm{C})$ and IgM line (M) appear on the test strip. 
Calculation of results for ELISA, the O.D was read at 450 $\mathrm{nm}$ with a microwell reader. The cut-off O.D $=2.1 \mathrm{x}$ negative control O.D. (if the O.D value of the negative control was lower than 0.05 , the calculation was done as per 0.05 ; if the O.D value was more than 0.05 , the calculation was done using the actual data).

Positive: sample O.D $\geq$ cut-off O.D. Negative: sample O.D <cut-off O.D.

\section{Statistical analysis}

The data recorded in the questionnaire and laboratory results were entered into the computer and analysed using a statistical package SPSS (version 21). To test the validity of RDT versus ELISA test as gold-standard, Cohen's Kappa Coefficient (k) with Kappa's cut-offs was used. $^{12}$ Diagnostic accuracy tests were used which included sensitivity, specificity, positive predictive value (PPV), negative predictive value (NPV), discriminating ability (DA), diagnostic efficiency and area under the curve (AUC) in order to validate the predictors. ${ }^{13}$

The associations between toxoplasmosis and possible risk factors were tested with Chi-square test. The magnitude of associations was assessed using odds ratios (OR) at 95\%CI. Multivariate logistic regression model was used to identify the explanatory variables among confounding risk variables that would explain the occurrence of toxoplasmosis. We used a backward stepwise selection model to select factors with a p-value of less than 0.1 to be fitted into the multivariate logistic regression analysis ${ }^{(14)}$. Factors with the $\mathrm{p}$-value $\leq$ less than or equal to 0.05 were considered to have a statistical significant association with T.gondii infection.

\section{RESULTS}

The overall seroprevalence of T.gondii infection among the pregnant women was $(11.3 \%)$ detected by RDT, significantly increased to $(57.9 \%)$ by ELISA test $(\mathrm{X} 2=$ 5.3; p=0.001) table (1). IgG (+ve) and IgM (-ve) which indicate a previous exposure were $(57.33 \%)$ and $(11.3 \%)$ among pregnant women by ELISA, and RDT respectively. The positive result of both $\operatorname{IgG}$ and $\operatorname{IgM}$ indicating primary infection or reactivation of chronic infection which was $(0.52 \%)$ has diagnosed by ELISA only. The negative results for both $\operatorname{IgG}$ and $\mathrm{IgM}$ indicating susceptible cases were $(42.2 \%)$ and $(88.7 \%)$ as diagnosed by ELISA and RDT respectively.

The diagnostic parameters of RDT versus ELISA as diagnostic gold standard showed that RDT had the sensitivity of $(15.8 \%)$, the specificity of (95\%), PPV of $(81.4 \%)$, NPV of $(45.1 \%)$, with overall diagnostic efficiency of $(49.2 \%)$. The DA was $(26.5 \%)$ and AUC was 0.554 table (2), fig (1).

Seroprevalence of T.gondii infection in relation to the history of exposure to different risk factors of T.gondii infection:.

Table 1: Seroprevalence of T.gondii infection among pregnant women according to the diagnostic methods used.

\begin{tabular}{|c|c|c|c|c|c|}
\hline \multirow[t]{2}{*}{$\begin{array}{l}\text { Diagnostic } \\
\text { methods }\end{array}$} & \multicolumn{2}{|c|}{$\begin{array}{l}\text { Examined } \\
\text { sample } \\
n=382\end{array}$} & \multirow[t]{2}{*}{$\begin{array}{l}\text { Percent } \\
\text { infected } \\
(\%)\end{array}$} & \multirow[t]{2}{*}{$\mathbf{X}^{2}{ }_{\mathrm{mc}}$} & \multirow[t]{2}{*}{$\mathbf{P}$} \\
\hline & $\begin{array}{l}\text { No } \\
\text { (-ve) }\end{array}$ & $\begin{array}{l}\text { No } \\
\text { (+ve) }\end{array}$ & & & \\
\hline ELISA & 161 & 221 & 57.9 & & \\
\hline RDT & 339 & 43 & 11.3 & 5.3 & $0.001^{*}$ \\
\hline
\end{tabular}

$\mathbf{X}^{\mathbf{2}} \mathbf{m c}=$ Mc-Nemar test; ${ }^{*}$ Significant at $\mathrm{p} \leq 0.05$

\section{In relation to sociodemographic characteristics}

The age of the participants ranged between 17-44 years with mean of $26.71 \pm 5.27$ years. The majority of participants were of age category $25-34$ years $(51.8 \%)$, followed by those aged between $15-24$ years $(38.7 \%)$. The highest seroprevalence was among those aged between $35-44$ years $(12.2 \%)$. The association between T.gondii infection and the age of the pregnant women was found to be statistically significant $(\mathrm{OR}=2.84$, 95\% CI $=1.251-6.455)$ i.e. the seroprevalence showed an increasing pattern with increasing age group. Those of age category between 35-44 years were 2.8 folds more likely exposed to infection than those of age category between 17-24 years.

Table 2: Diagnostic parameters of RDT versus ELISA.

\begin{tabular}{|c|c|c|c|c|c|c|c|}
\hline & $\begin{array}{l}\text { Diagnostic } \\
\text { efficiency \% }\end{array}$ & $\begin{array}{l}\text { Sensitivity } \\
\%(95 \% \mathrm{CI})\end{array}$ & $\begin{array}{l}\text { Specificity } \\
\%(95 \% \text { CI) }\end{array}$ & $\begin{array}{l}\text { PPV } \\
\%(95 \% \mathrm{CI})\end{array}$ & $\begin{array}{l}\text { NPV } \\
\%(95 \% \mathrm{CI})\end{array}$ & DA \% & $\begin{array}{l}\text { AUC } \\
(95 \% \text { CI })\end{array}$ \\
\hline $\begin{array}{l}\text { Toxoplasma } \\
\text { by RDT }\end{array}$ & 49.2 & $\begin{array}{l}15.8 \\
(11.4-21.5)\end{array}$ & $\begin{array}{l}95.0 \\
(90.1-97.7)\end{array}$ & $\begin{array}{l}81.4 \\
(66.1-91.1)\end{array}$ & $\begin{array}{l}45.1 \\
(40.0-50.6)\end{array}$ & 26.5 & 0.554 \\
\hline
\end{tabular}

PPV: Positive predictive value; NPV: Negative predictive value; DA: Discrimination ability; AUC: Area under the curve; RDT: Rapid diagnostic test; CI: Confidence intervals. 
With regard to occupation and level of education, the association was not statistically significant. The association between residences of the pregnant women and sero-positivity was not statistically significant.

\section{In relation to animal rearing factors}

Only $5 \%$ of the infected pregnant women had cats in their households. The majority of infected cases $(90.9 \%)$ were feeding their cats with table food. Also (54.5\%) of them were removing the cat's feces without using gloves and $(12.5 \%)$ of them didn't wash their hands after removing the cat's feces. Twenty six $(11.8 \%)$ of seropositive pregnant women were rearing other animals as birds (chickens or ducks) in their households. There was no significant relationship between the sero-positive pregnant women and animal rearing risk factors.

Table 3: Seroprevalence of T.gondii infection in relation to obstetric variables among the studied sample.

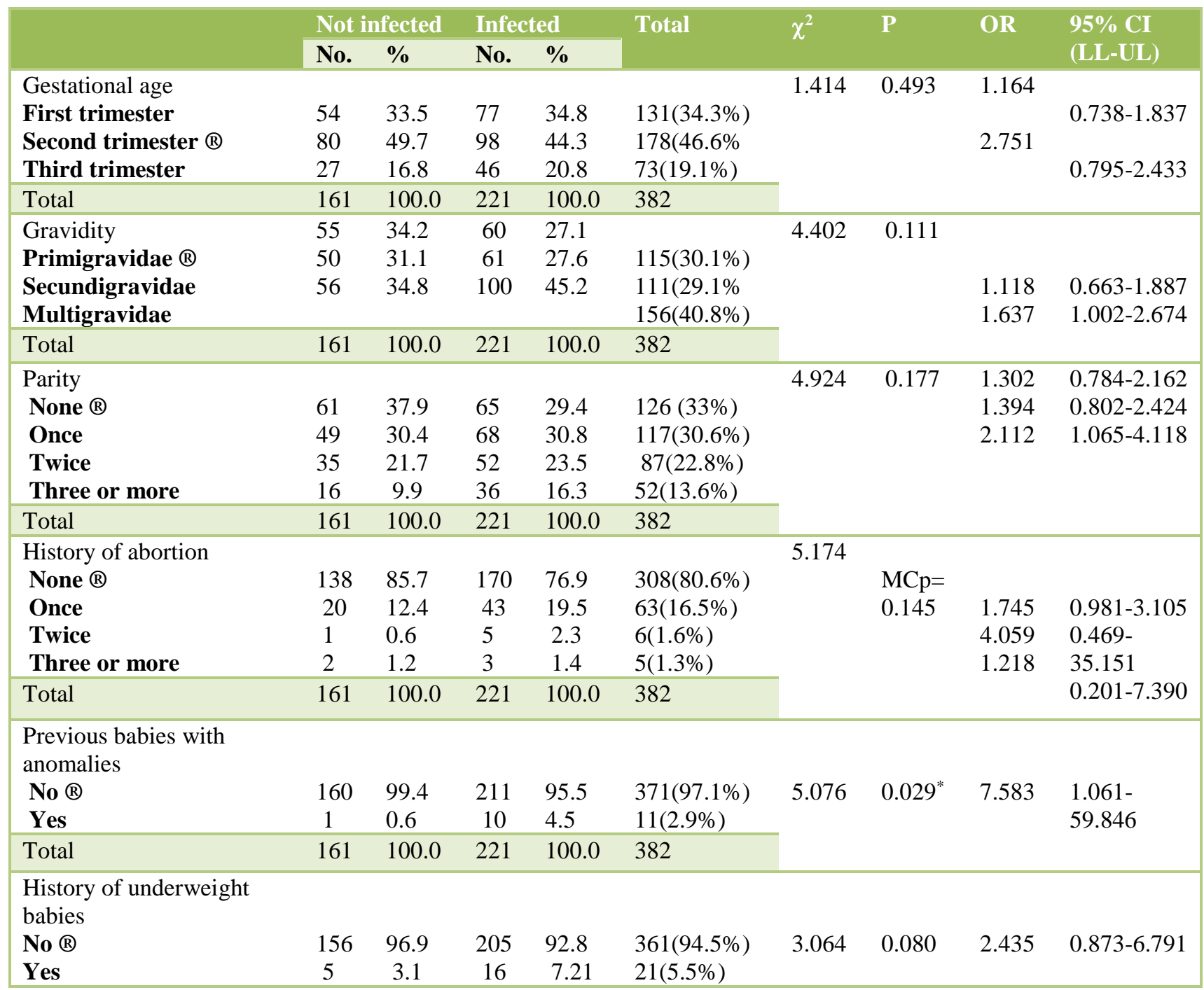

$\chi^{2:}$ Chi square test MC: Mont Carlo test; OR: Odds ratio CI: Confidence interval LL: Lower limit UL: Upper limit; *Statistically significant at $\mathrm{p} \leq 0.05$.

\section{In relation to Kitchen hygiene and ways of cooking}

The majority of the seropositive pregnant women $(96.4 \%)$ were eating well done meat. Only (34.8\%) were eating raw vegetables outdoors. Most of them (98.6\%) were washing their vegetables before eating. The percentage of the infected pregnant women who were washing vegetables before eating by using running tap water was $(37.6 \%)$ or rinse in the collected water was $(42.2 \%)$. Only $(20.2 \%)$ of them were rinsing their vegetables in water with vinegar.

Most of the infected pregnant women were washing their hands and utensils before cooking (73.8\% and 67.9\%) 
respectively. About (68\%) of the seropositive pregnant women were washing the knives before cooking and about $(85 \%)$ of them using the same knife for cutting vegetables and meat.

About (47\%) of the seropositive pregnant women were using a cutting board. Only (13.5\%) of them were using separate cutting board for cutting vegetables and meat. The majority of infected participants $(98.9 \%)$ were washing the cutting board before use. About (67\% and $69 \%$ ) of the infected participants were consuming processed meat and fresh milk respectively. There was no statistically significant relationship between T.gondii infection and any of these risk variables.

Table 4: Multivariate logistic regression analysis of risk factors for $\mathbf{T}$.gondii infection among the studied sample.

\begin{tabular}{|llllll|}
\hline & B & Sig. & OR & \multicolumn{2}{l|}{$95 \%$ CI } \\
\hline Age & 0.046 & $0.029^{*}$ & 1.047 & 1.005 & 1.091 \\
\hline $\begin{array}{l}\text { Meat } \\
\text { cooking }\end{array}$ & 0.682 & 0.151 & 0.506 & 0.199 & 1.283 \\
\hline $\begin{array}{l}\text { Using same } \\
\text { knife for } \\
\text { cutting } \\
\text { vegetables } \\
\text { and meat }\end{array}$ & 0.531 & 0.054 & 1.701 & 0.990 & 2.924 \\
\hline $\begin{array}{l}\text { History of } \\
\text { underweight } \\
\text { babies }\end{array}$ & 0.390 & 0.509 & 1.477 & 0.464 & 4.696 \\
\hline $\begin{array}{l}\text { History of } \\
\text { abnormal } \\
\text { babies }\end{array}$ & 1.733 & 0.122 & 5.659 & 0.630 & 50.820 \\
\hline
\end{tabular}

Classification accuracy of the model 61\%; OR: Odds ratio; CI: Confidence interval; LL: Lower limit; UL: Upper limit; $*$ Statistically significant at $\mathrm{p} \leq=0.05$.

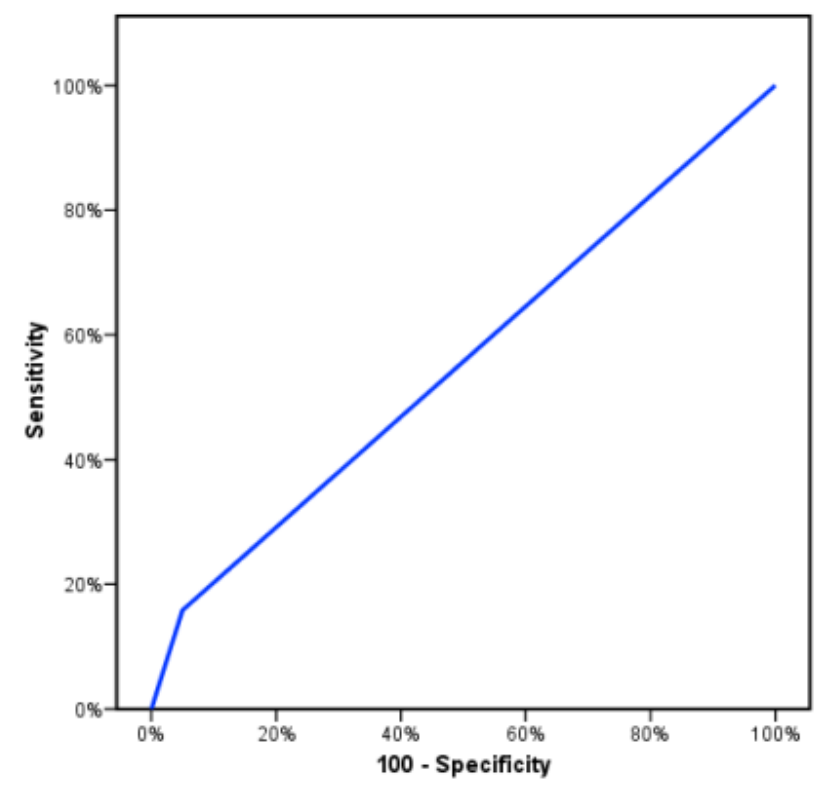

Figure 1: The area under the ROC curve (AUC). Sensitivity and specificity of RDT versus ELISA.

\section{In relation to obstetric variables}

Table 3 illustrates the seroprevalence of $T$. gondii infection in relation to obstetric variables among the studied sample. The association between the history of delivering babies with congenital anomalies and the T.gondii infection was found to be statistically significant $(\mathrm{OR}=7.6,95 \% \mathrm{CI}=1.061-59.846)$ i.e. pregnant women who had the history of delivered abnormal babies were more likely exposed to infection 7.6 folds than those who hadn't have this history.

\section{In relation to the knowledge about toxoplasmosis}

The majority of the seropositive participants (94.6\%) had no knowledge about toxoplasmosis as a disease. In addition, (25.0\%) of them had no knowledge about the routes of transmission of toxoplasmosis and (33.3\%) had no knowledge about the methods of prevention. But these factors didn't show any statistic difference between infected and non-infected participants.

In multivariate analysis (table 4), stepwise logistic regression technique was used and the relative effect of the independent variable on the outcome variable was determined. Only variables that reached a p-value less than 0.1 were taken in this analysis. These factors were age, meat cooking, using same knife for cutting vegetables and meat, history of previous low birth weight babies and history of babies with congenital anomalies in previous deliveries. Age $(\mathrm{OR}=2.84,95 \% \mathrm{CI}=1.251-6.455)$ remained significant in the final step of multivariate analysis, but history of previous deliveries of babies with congenital anomalies that was significantly associated with the seropositivity of T.gondii infection turned to be insignificant after it was adjusted for other explanatory variables. Other factors including, meat cooking, using the same knife for cutting vegetables and meat, history of previous low birth weight babies, didn't correlate significantly with toxoplasma infection risk in these study participant. The classification accuracy of the model was $61 \%$ of cases.

\section{DISCUSSION}

Toxoplasmosis is usually asymptomatic in immunocompetent hosts and induces a self-limiting disease. However, infection in pregnant women can cause serious health problems for the fetus. If T. gondii infects a pregnant woman with no previous exposure to the parasite, it can migrate through the placenta into the fetus and subsequently cause congenital toxoplasmosis. ${ }^{15}$ The diagnosis of toxoplasmosis during pregnancy relays on serology. 
In a view of the worldwide importance of T.gondii and the limited data of the seroprevalence of this parasite among pregnant women in Alexandria, Egypt, the present study was done.

In the present study, two diagnostic techniques were used, Rapid diagnostic test (RDT) or the immunochromatographic test (ICT) and ELISA.

Rapid diagnostic test (RDT) or the immunochromatographic test (ICT) was first developed in the late 1960s mainly for the detection of serum proteins. The test uses recombinant truncated surface antigen for detection of antibodies against T.gondii. It is rapid, and easy to perform and interpret. Generally, a higher concentration and purity of the antigen are required for this test. ${ }^{16}$

ELISA is an accurate serological tool for the diagnosis of toxoplasmosis depending on antigen-antibody interaction. The reaction can be assessed objectively by quantization of the color that developed by an ELISA reader. The procedure is simple to perform, economical and easily adoptable for field use. ELISA is also suited for laboratories required to analyze large number of samples. $^{17}$

In our study, RDT was able to detect only 43 pregnant women to be seropositive for T.gondii, while ELISA technique detected 221 positive cases, 186 of them were diagnosed negative by RDT. These results showed a slight Cohen's Kappa agreement $(\mathrm{K}=0.094)$ between RDT and ELISA techniques for the diagnosis of T. gondii infection. RDT had the sensitivity of (15.8\%) which indicated that the RDT was able to detect only $(15.8 \%)$ from pregnant women who were infected by T.gondii. the specificity of $(95 \%)$ which indicated that the RDT was able to detect a proportion of pregnant women (95\%) who were not infected.

In agreement with our results, Abd elkareem et al, found that out of 96 cases; ELISA could detect $(27.1 \%)$ to be seropositive for IgG, while ICT detected only (18.8\%). According to these results ELISA IgG was found more sensitive than ICT. ${ }^{18}$

On the contrary to our results, Huang et al, studied the results of using ICT in detecting anti-T.gondii antibodies in cats sera comparing with ELISA results. ${ }^{19}$ The relative sensitivity and specificity of ICT were 97.2 and $95.8 \%$. The relative agreement was $96.1 \%$ between the ICT and ELISA. These high agreements suggested that ICT would be more sensitive than the ELISA in their study.

Again, Hassan et al, showed a higher sensitivity (88\%) and lower specificity $(89.29 \%)$ than our results in detecting IgG antibodies, when studied latex agglutination test and ELISA for diagnosis of toxoplasmosis. ${ }^{20}$
Terkawi et al, developed ICT based on recombinant dense granules antigen protein derived from T.gondii (TgGRA7) for rapid detection of antibodies to the infection. $^{21}$ A total 59 serum sample from pigs were examined with ICT and compared with those with ELISA. The sensitivity and specificity of ICT was $72.7 \%$ and $100 \%$ respectively with a Kappa value of 0.70 (substantial agreement).

In our study, RDT had PPV of $(81.4 \%)$ which means that the RDT had the ability to detect about $(81 \%)$ of those who have toxoplasmosis among those whose test results were positive. NPV of $(45.1 \%)$ denoting that RDT was able to detect about (45\%) of those who didn't have the disease among those whose test results were negative. By performing the ROC curve and estimating AUC $(0.554 \%)$ which indicated a bad diagnostic parameter. Besides the discrimination ability DA of $(26.5 \%)$ indicating that RDT was a bad diagnostic test with diagnostic efficiency of (49.2\%).

The seroprevelanve in the present study was higher than those reported in other countries that studied the prevalence of T.gondii among pregnant women e.g. Nijem et al, the overall seroprevalence of IgG antibodies to T. gondii was (27.9\%). ${ }^{22}$ In Europe, Cvetkovic et al, studied the prevalence of Toxoplasma infection in pregnant women in a series of 235 pregnant women using ELISA technique showed that the overall prevalence was $(20.4 \%){ }^{23}$ Sakikawa et al, in Japan using latex agglutination; the overall prevalence was $(10.3 \%) .{ }^{24}$ Flatt et al, in London sero-prevalence was (17.32\%). ${ }^{25}$ Aqeely et al, studied the seroepidemiology in Jazan province, Saudi Arabia using ELISA technique. ${ }^{26}$ The overall seroprevalence among the studied sample were $(24.1 \%)$.

In this study, the association between T.gondii infection and the age of the pregnant women was found to be statistically significant $(\mathrm{OR}=2.84,95 \% \mathrm{CI}=1,251$ 6.455). Corroborating with our work, Nassef et al and Almushait et al. ${ }^{27,28}$ In the multivariate analysis, only age category remained significant in the final step of multivariate analysis.

In Brazil by Sroka et al, multivariate regression analysis showed that there are some variables which identified as a risk factors by the bivariate analysis turned to insignificant such as age, low monthly income, living in poor housing conditions, including streets made of sand and houses with sand floor and consumption of powder milk, while others that weren't significant by the bivariate analysis turned to significant as consumption of vegetables washed by untreated water. ${ }^{29}$

Al-Eryani et al, studied the factors associated with high seroprevalence of T.gondii infection among pregnant women in Yemen. ${ }^{30}$ Bivariate analysis identified the following factors to be significantly associated with toxoplsmosis (IgG and/or IgM): age $\geq 25$ years, education level, number of children, cat rearing, contact with soil, 
use of unwashed kitchen knife, and eating unwashed vegetables. While the independent factors in the multivariate logistic regression analysis included age $\geq 25$ years, cat rearing, and contact with soil.

Andiappan et al, in southern Thailand, for univariate analysis, the study verified that age group, occupation and source of drinking water had significant associations with seropositive pregnant women. ${ }^{31}$ After the multivariate logistic regression analysis confirmed that age $\geq 26$, working as a laborer and drinking unclean (piped/tap/rain) water were identified as significant risk factors for Toxoplasma acquisition.

\section{CONCLUSION}

The present study has documented a bad diagnostic performance of RDT in detection T.gondii in serum samples of infected pregnant women as compared to ELISA technique. Although it is relatively low-priced and less time consuming, RDT is not appropriate diagnostic methods for screening of the disease or surveillances program. Age is the only risk factor to be associated statistically with toxoplasma gondii infection.

Funding: No funding sources

Conflict of interest: None declared

Ethical approval: The study was approved by the Institutional Ethics Committee

\section{REFERENCES}

1. Dubey JP. The history of Toxoplasma gondii - the first 100 years. J Eukaryot Microbiol. 2008;55(6):467-75.

2. Dubey JP. The discovery of the life cycle of Toxoplasma gondii. Int J Parasitol. 2009;39(8):87782.

3. Periera KS, Franco RM, Leal DA. Transmission of toxoplasmosis (Toxoplasma gondii) by foods. Adv Food Nutr Res. 2010;60:1-19.

4. Flegr J, Priplatova L, Hampl R, Bicikovia M, Ripova D, Mohr P. Difference of neuro-and immunomodulatory steroids and Selected hormone and lipid concentration between Toxoplasma-free and Toxoplasma-infected but not CMV-free and CMV-infected schizophrenia patients. Neuro Endocrinal Lett. 2014;35(1):20-7.

5. Durlach RA, Kaufer F, Carral L, Hirt J. Toxoplasmic lymphadenitis-clinical and serologic profile. Clin Microbiol Infect. 2003;9(7):625-31

6. Dubey JP, Jones JL. Toxoplasma gondii infection in humans and animals in the United States. Int $\mathrm{J}$ Parasitol. 2008;38(11):1257-78.

7. Montoya JG, Rosso F: Diagnosis and management of Toxoplasmosis. Clin Perinatal. 2005;32:705-26.

8. Kravetz J. Congenital toxoplasmosis. Clinical Evidence. 2013;1:33-6.
9. Gomez-Marin, J. E. Congenital toxoplasmosis in South American children. Scientia Medica (Porto Alegre). 2010;20(6):103-7.

10. Naing L, Winn T, Rusil BN. Practical issues in calculating sample size for prevalence studies. Arch Orofac Sci. 2006;1:9-14.

11. El-Gozamy BR, Mohamed SA, Mansour HA. Toxoplasmosis among pregnant women in Qualyobia governorate, Egypt. J Egypt Soc Parasitol. 2009;39(2):389-401.

12. Landis JR, Koch GG. The measurement of observer agreement for categorical data. Biometrics. 1977;33(1):159-74.

13. Eusebi P. Diagnostic accuracy measures. Cerebrovasc Dis. 2013;36:267-72.

14. Victoria CG, Huttly SR, Fuchs SC, Olinto MT. The role of conceptual frameworks in epidemiological analysis: a hierarchical approach. Int J Epidemiol, 1997;26:224-7.

15. Jones J, Lopez A, Wilson M. Congenital toxoplasmosis. Am Fam Pysician. 2003;67(10):2131- 8 .

16. El-Moamly AA. Immuno-chromatographic Techniques: Benefits for the Diagnosis of Parasitic Infections. Austin Chromatogr. 2014;1(4):1-8.

17. Sudan V, Jaiswal AK, Shanker D. Recent trends in the diagnosis of toxoplasmosis. Clin Rev Opinions. 2013;5(2):11-7.

18. Abd elkareem BA, Eltayeb MM. Seropositivity of toxoplasmosis in Sudanese males blood donors in militarie hospital Omdurman, Sudan. IJB. 2015;6(3):147-9.

19. Huang X, Xuan X, Hirata H, Yokoyama N, Xu L, Suzuki N, et al. Rapid immunochromatographic test using recombinant SAG 2 for detection of antibodies againist Toxoplasma gondii in cats. J Clin Microbiol. 2004;42(1):351-3.

20. Hassan JS, Ghazi HF, Ahmed AAH. Evaluation of rapid chromatographic immunoassay with latex agglutination test and (ELISA) for diagnosis of human toxoplasmosis. Fac Med Paghdad. 2010;52(4):468-70.

21. Terkawi MA, Kameyama K, Rasul NH, Xuan X, Nishikawa Y. Development of an Immunochromatographic Assay Based on Dense Granule Protein 7 for Serological Detection of Toxoplasma gondii Infection. Clin Vaccine Immunol. 2013;20(4):596-601.

22. Nijem KI, Al-Amleh S. Seroprevalence and associated risk factors of toxoplasmosis in pregnant women in Hebron district, Palestine. EMHJ. 2009; 15(5):1278- 84.

23. Cvetkovik D, Bobic B, Jankovska G, Klun I, Panovski N, Djurkovic-Djakovic O. Risk factors of Toxoplasma infection in pregnant women in FYR of Macedonia. Parasite. 2010;17:183- 6.

24. Sakikawa M, Noda S, Hanaoka M, Nakayama H, Hojo S, Kakinoki S, et al. Anti-Toxoplasma antibodies prevalence, primary infection rate, and risk factors in a study of toxoplasmosis in 4,466 
pregnant women in Japan. Clin Vaccin Imunol. 2012;19(3):365-7.

25. Flatt A, Shetty N. Seroprevalence and risk factors for toxoplasmosis among antenatal women in London: a re-examination of risk in an ethnically diverse population. Eur J Public Health. 2013;23(4):648-52.

26. Aqueely H, El-Gayar EK, Perveen Khan D, Najmi A, Alvi A, Bani I, et al. Seroproepidemiology of Toxoplasma gondii among pregnant women in Jazan Province, Saudi Arabia. J Trop Med. 2014.

27. Nassef NE, Abd El-Ghaffar MM, El-Nahas NS, Hassanain ME, Shams El-Din SA, Ammar AI. Seroprevalence and genotyping of Toxoplasma gondii in Menufia governorate. Menufia Med J. 2015;28(3):617-27.

28. Almushait MA, Bin Dajem SM, Elsherbiny NM, Eskandar MA, Al Azraqi TA, Makhlouf LM. Seroprevalence and risk factors of Toxoplasma gondii infection among pregnant women in south western, Saudi Arabia. J Parasit Dis. 2014;38(1):410.
29. Sroka S, Bartelheimer N, Winter A, Heukelbach J, Ariza L, Ribeiro H. Prevalence and Risk Factors of Toxoplasmosis among Pregnant Women in Fortaleza, Northeastern Brazil. Am J Trop Med Hyg. 2010;83(3):528-33.

30. Al-Eryani SMA, Al-Mekhlafi AM, Al-Shibani LA, Mahdy MMK, Azazy AA. Toxoplasma gondii infection among pregnant women in Yemen: Factors associated with high seroprevalence. J Infect Dev Ctries. 2016;10(6):667-72.

31. Andiappan H, Nissapatorn V, Sawangjaroen N, Chemoh W, Lau YL, Kumar T, et al. Toxoplasma infection in pregnant women: a current status in Songklanagarind hospital, southern Thailand. Parasit Vectors. 2014;7:239.

Cite this article as: Bassiony HK, Soliman NK, El Tawab S, Eassa SM, Eissa A. Sero-prevalence and risk factors associated with toxoplasma gondii infection among pregnant women in Alexandria, Egypt. Int J Reprod Contracept Obstet Gynecol 